\title{
Akutes Koronarsyndrom ohne ST-Hebung - Diagnostik
}

\section{Weber}

C. Hamm

\section{Acute coronary syndromes without ST-elevation - diagnosis}

In westlichen Ländern steht die koronare Herzkrankheit (KHK) an erster Stelle der Todesursachenstatistik. Die Häufigkeit wird auch in Zukunft weiter zunehmen. Diagnostik und Therapie der KHK haben daher in der täglichen Routine einen sehr hohen Stellenwert.

Das klinische Bild der KHK variiert stark. Es kann zwischen einer chronischen Phase mit stabiler Beschwerdesymptomatik bei Belastung und einer akuten, lebensbedrohlichen Phase mit plötzlich auftretenden Beschwerden oder rasch progredienter Beschwerdesymptomatik, dem so genannten akuten Koronarsyndrom (ACS) unterschieden werden. Die Letalität liegt bei Patienten mit ACS nach dem Euro Heart Survey (6) und dem GRACE-Register (3) bei ca. $12 \%$ innerhalb von 6 Monaten und ist damit sehr hoch.

Das ACS wird durch eine akute thorakale Schmerzsymptomatik in Ruhe oder bei geringster Belastung charakterisiert. Es umfasst die instabile Angina pectoris, den Myokardinfarkt ohne ST-Streckenhebung (NSTEMI) und den klassischen Myokardinfarkt mit STStreckenhebung (STEMI) (Abb.1). Nach der neuen Infarktdefinition ist jede Troponinerhöhung als Myokardinfarkt zu verstehen (1).

Im Folgenden sollen Diagnostik und Therapie des ACS ohne STStreckenhebung dargestellt werden. Dabei werden die Empfehlungen („Task Force Report“) der europäischen kardiologischen Gesellschaft (ESC) berücksichtigt (2).

\section{Pathophysiologie}

Ausgangspunkt der KHK ist die atherosklerotische Plaque, die sich meist als Folge einer initialen Endothelläsion bildet und aus einem lipidreichen Kern mit einer fibrösen Kappe besteht. Je nach Aufbau und Zusammensetzung kann zwischen einer vulnerablen und einer stabilen Plaque unterschieden werden. Bei der chronischen KHK mit stabiler Angina Pectoris-Symptomatik bei Belastung führt die Lumeneinengung durch eine stabile Plaque zur Behinderung des Blutflusses. Dagegen kommt es beim ACS zur Ruptur oder Erosion einer vulnerablen Plaque mit Thrombusbildung. Dieser Thrombus kann zum kompletten Gefäßverschluss führen. Dies ist die Ursache eines akuten ST-Hebungsinfarktes. Es kann aber auch

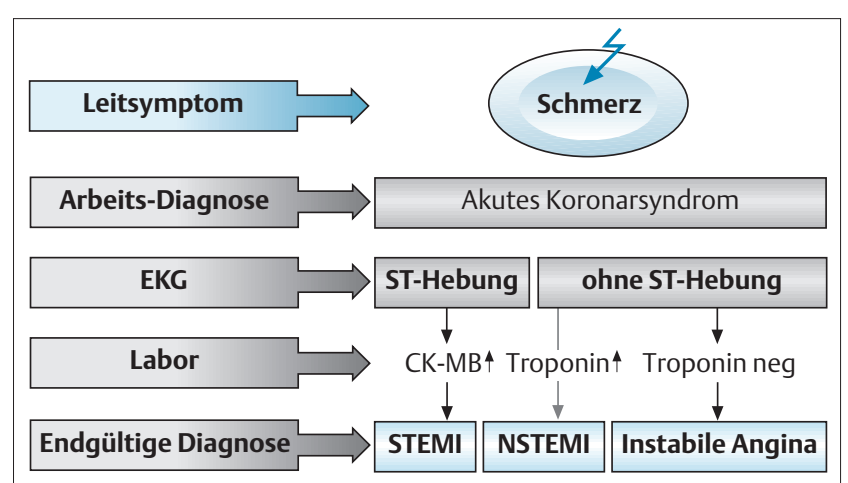

Abb.1 Schematische Darstellung des akuten Koronarsyndroms. STEMI, (NSTEMI): akuter Myokardinfarkt mit (ohne) ST-Hebungen ([Non] ST-Elevation Myocardial Infarction).

durch wiederholte Embolisation thrombotischen Materials in die peripheren Koronarien zur instabilen Angina pectoris oder zu einem Myokardinfarkt ohne ST-Streckenhebungen kommen. Als Folge der endogenen Fibrinolyse ist auch ein Wechsel zwischen Okklusion und subtotaler Okklusion mit entsprechendem fluktuierenden klinischen Verlauf möglich.

\section{Symptomatik}

Das Kardinalsymptom des ACS ist der Thoraxschmerz (Angina pectoris). Dieser wird meist als Druckschmerz, Engegefühl oder als Brennen empfunden. Eine Schmerzausstrahlung in den linken Arm, den Hals, den Kiefer oder den Rücken ist typisch. Begleitend zur Schmerzsymptomatik kann Dyspnoe und auch eine vegetative Symptomatik bestehen. Die Beschwerden können sowohl akut aus der Ruhe heraus als auch belastungsabhängig auftreten. Atypische Beschwerden treten gelegentlich bei jungen Patienten (25-40 Jahre), älteren Patienten (> 75 Jahre) und bei Diabetikern auf. Einziges Symptom kann dann Dyspnoe, epigastrischer Schmerz oder pleuritischer Schmerz sein, jedoch können die Patienten auch komplett asymptomatisch bleiben (stumme Ischämie). Zur Beschreibung der Angina pectoris hat 
sich in der Praxis die CCS-Klassifikation (Canadian Cardiac Society) durchgesetzt (Tab.1).

Die Diagnose „Instabile Angina pectoris“ wird aufgrund des klinischen Bildes gestellt, die Einteilung erfolgt nach der BraunwaldKlassifikation. Entgegen früherer Definitionen wird diese Diagnose heute nur bei Patienten mit Thoraxschmerz in Ruhe oder bei geringster Belastung (CCS IV) gestellt, die innerhalb der letzten 48 Stunden aufgetreten sind (Braunwald Klasse IIIb).

\section{Diagnostik und Risilkostratifizierung}

Ziel der diagnostischen Maßnahmen ist es, die klinische Verdachtsdiagnose des ACS durch objektive Befunde zu erhärten oder auszuschließen und eine Risikostratifizierung vorzunehmen, die im Wesentlichen auf EKG und biochemischen Markern beruht und Grundlage der weiteren Therapie ist. Aufgrund des zu ermittelnden individuellen Risikos des Patienten muss die Entscheidung getroffen werden, wo (ambulant/stationär) und wie (medikamentös/interventionell) die Behandlung erfolgt.

\section{EKG}

Erste diagnostische Maßnahme bei Patienten mit akutem Thoraxschmerz ist das Anfertigen eines Ruhe-EKGs, um einen Myokardinfarkt mit ST-Streckenhebung auszuschließen. Bei fehlenden ST-Hebungen sind die EKG-Veränderungen, die beim ACS auftreten, vielgestaltig und oft unspezifisch. So ist bekannt, dass etwa 55\% der Patienten mit ACS entweder ein normales oder ein diagnostisch nicht verwertbares EKG (Schrittmacher-EKG, Schenkelblock) haben. Nur bei etwa $20 \%$ finden sich ST-Streckensenkungen und bei etwa $25 \%$ negative T-Wellen (4). Diese Veränderungen des ST-T-Segments können durch eine akute Myokardischämie, aber auch durch andere kardiale Erkrankungen bedingt sein. Das EKG ist somit nicht geeignet, die Diagnose des ACS zu sichern, es hat aber Bedeutung als prognostischer Marker. Aus zahlreichen Studien ist gut validiert, dass Patienten mit einem ACS und ST-Streckensenkung $>1 \mathrm{mV}$ in mindestens zwei Ableitungen ein deutlich erhöhtes Risiko haben. Die Ereignisrate für Tod kardialer Ursache und Myokardinfarkt liegt bei diesen Patienten bei $10-12 \%$ innerhalb von 30 Tagen. Dagegen haben negative T-Wellen eine ähnliche prognostische Wertigkeit wie ein normales EKG mit einer Ereignisrate zwischen 2\% und 4\% für Tod und Myokardinfarkt $(4,9)$.

kurzgefasst: ST-Streckensenkungen>1 $\mathbf{~ m V}$ im Ruhe-EKG sind ein guter Prädiktor für ein erhöhtes Risiko bei Patienten mit ACS. Patienten mit T-Wellenveränderungen haben dagegen kein wesentlich erhöhtes Risiko.

\section{Biochemische kardiale Marker}

Laborchemische Methoden werden üblicherweise herangezogen, um akuten Thoraxschmerz bei Patienten besser zu beurteilen. Die Bestimmung der Kreatinkinase (CK) und des Isoenzyms CK-MB ist seit mehr als 30 Jahren der „Goldstandard“ zur Erkennung einer Myokardzellschädigung. Ein transmuraler und nicht-transmuraler Myokardinfarkt lässt sich damit diagnostizieren. Von histopathologischen Untersuchungen wissen wir allerdings, dass kleinere Myokardzellschädigungen, die beim ACS eine wichtige pathogenetische Rolle spielen, durch die CK-MB-Bestimmung nicht entdeckt werden. Als neue biochemische Marker haben sich die Troponine etabliert und eine zentrale Stellung in den europäischen und amerikanischen Empfehlungen erhalten.
Tab.1 CCS - Klassifikation der Angina pectoris (Canadian Cardiac Soc.).

\begin{tabular}{lll}
\hline Grad & Definition & Beispiel \\
\hline I & $\begin{array}{l}\text { Keine Angina bei normaler } \\
\text { Belastung. Angina bei sehr } \\
\text { hoher Belastung oder } \\
\text { andauernder Anstrengung }\end{array}$ & Gartenarbeit, Ballsportarten \\
II & $\begin{array}{l}\text { Geringe Einschränkung bei } \\
\text { normalen Tätigkeiten }\end{array}$ & $\begin{array}{l}\text { Angina beim schnellen Treppenstei- } \\
\text { gen (> 1 Etage), beim Bergaufgehen }\end{array}$ \\
III & $\begin{array}{l}\text { Deutliche Einschränkung } \\
\text { der Leistungsfähigkeit }\end{array}$ & $\begin{array}{l}\text { Angina beim An- und Ausziehen, } \\
\text { Treppensteigen (<1 Etage), Gehen in } \\
\text { der Ebene, leichter Hausarbeit }\end{array}$ \\
IV & $\begin{array}{l}\text { Angina bei geringster } \\
\text { Belastung oder in Ruhe }\end{array}$ & \\
\hline
\end{tabular}

Der Troponinkomplex reguliert die elektromechanische Koppelung der Myofibrillen und setzt sich aus drei Untereinheiten, dem Troponin T, I und C zusammen. Die kardialen Isoformen von Troponin $\mathrm{T}$ und I finden sich nicht in der Skelettmuskulatur, und es besteht auch keine Kreuzreaktivität zu skelettmuskulären Formen. Ihr Nachweis gelingt heute quantitativ durch gut standardisierte Assays auf modernen Analyseautomaten mittels ELISA-Technik. Zusätzlich stehen qualitative Testsysteme (positiv/negativ) zur Verfügung, die auch als bettseitige Tests durchgeführt werden können und innerhalb von 15-20 min zuverlässige Ergebnisse liefern. Die Bestimmung der Troponine hat sich deshalb heute als neuer „Goldstandard“ zur Erkennung von Herzmusklschädigungen etabliert.

Troponine sind im Blut von Gesunden nicht oder nur in äußerst niedrigen Konzentrationen nachweisbar, so dass jede Erhöhung als pathologisch zu werten ist. Zur Troponinerhöhung kommt es bereits nach myokardialen Mikrozellschädigungen, bei denen es zu keinem Anstieg der CK und der CK-MB kommt. Diese Zellschädigung beruht nach heutigem Wissen auf einer Embolisation thrombotischen Materials nach Plaqueruptur in einem größeren epikardialen Gefäß. Die Troponinerhöhung ist frühstens 3-4 Stunden nach einem entsprechenden Ereignis zu beobachten. Troponinanstiege können auch bei Herzmuskelschädigungen anderer Genese vorkommen. So finden sich Troponinanstiege auch bei Myokarditis, akutem Linksherzversagen, akuter Lungenembolie und bei hypertensiven Krisen. Bei Patienten mit präterminaler oder dialysepflichtiger Niereninsuffizienz finden sich erhöhte Troponinspiegel auch ohne dass ein ACS besteht. Allerdings haben Troponine auch bei Patienten mit Niereninsuffizienz eine prognostische Aussagekraft.

Bei etwa 1/3 der Patienten mit akutem Thoraxschmerz können erhöhte Troponin-T- oder -I-Werte nachgewiesen werden, ohne dass die CK-MB über dem Normbereich liegt. In großen multizentrischen Studien wurde in den letzten Jahren dokumentiert, dass der Nachweis von zirkulierenden Troponinen mit einem erhöhten Risiko für die Patienten assoziiert ist (5) und dass eine Troponinbestimmmung bei Aufnahme und eine zweite Bestimmung 6-12 Stunden später eine überlegene Methode zur Risikostratifizierung ist (4). Eine Einzelbestimmung ist nicht ausreichend, da etwa $20 \%$ der Patienten mit positivem Troponin erst bei der Zweitbestimmung erkannt werden.

In jüngster Zeit haben inflammatorische Marker zunehmend an Bedeutung gewonnen. Als inflammatorischer Marker ist das Creaktive Protein (CRP) am besten untersucht. Durch Einführung hochsensitiver Assays (hsCRP) gelingt der Nachweis auch 
geringer CRP-Erhöhungen zuverlässig. Eine Erhöhung des hsCRP bei Patienten mit ACS ist verbunden mit einem erhöhten Risiko für Tod und Myokardinfarkt $(7,8)$. Im Unterschied zu den Troponinen, die das akute Risiko reflektieren, ist das hsCRP ein guter Marker für das Langzeitrisiko.

kurzgefasst: Die Troponinerhöhung bei Patienten mit ACS ist verursacht durch myokardiale Mikroinfarkte. Diese entstehen als Folge peripherer Embolisation thrombotischen Materials bei Ruptur einer vulnerablen Plaque. Die Troponine haben sich als überlegener Marker zur Risikostratifizierung bei Patienten mit einem ACS erwiesen.

\section{Echokardiographie}

Die linksventrikuläre systolische Funktion ist eine wichtige prognostische Größe bei Patienten mit KHK und kann einfach und zuverlässig echokardiographisch ermittelt werden. Es können passagere oder dauerhafte regionale Wandbewegungsstörungen erfasst werden. Dies erlaubt indirekte Aussagen über die Lokalisation und hämodynamische Relevanz einer Koronarläsion. Andere differentialdiagnostisch wichtige kardiale Erkrankungen wie Klappenvitien, angeborene Fehlbildungen oder Kardiomyopathien können erkannt werden. Der echokardiographische Befund ist aus diesem Grunde für die weitere Therapieentscheidung sehr wichtig.

\section{Belastungsuntersuchungen}

Bei allen Patienten mit einer thorakalen Beschwerdesymptomatik, bei denen aufgrund der Risikostratifizierung keine Katheterintervention in der Akutphase erfolgt ist, ist eine Belastungsuntersuchung notwendig. In erster Linie sollte ein Belastungs-EKG angefertigt und dieses gegebenenfalls durch bildgebende Verfahren wie die Myokardszintigraphie, die Stressechokardiographie oder neuerdings auch das Stress-MRT ergänzt werden. Bei pathologischem Ergebnis mit positivem Ischämienachweis ist eine Neubewertung der Therapie erforderlich und dann die Indikation zur Koronarangiographie zu stellen.

\section{Koronarangiographie}

Anhand der Koronarangiographie kann der Koronarstatus ermittelt und somit eine exakte Diagnose gestellt werden. Dies ist Voraussetzung für die weitere invasive Therapie, da nur durch den angiographischen Befund über revaskularisierende Maßnahmen, Katheterintervention oder Bypassoperation, entschieden werden kann. Im Rahmen einer frühinvasiven Therapiestrategie bei Hochrisikopatienten (s.u.) sollte die Koronarangiographie frühzeitig, d.h. innerhalb der ersten 48 Stunden, erfolgen. Bei Patienten, die primär konservativ behandelt wurden, sollte eine Koronarangiographie immer dann durchgeführt werden, wenn erneut Beschwerden auftreten oder wenn sich in einer Belastungsuntersuchung Ischämiezeichen finden.

\section{Algorithmus zur Risilkostratifizierung}

Die erste diagnostische Maßnahme bei Patienten mit akut auftretenden thorakalen Schmerzen ist das EKG. Zeigt dieses ST-Streckenhebungen, so ist von einem Myokardinfarkt auszugehen und es muss eine entsprechende revaskularisierende Therapie, Thrombolyse oder Katheterintervention eingeleitet werden. Laborchemische Marker spielen für die Akutbehandlung keine Rolle und es sollte durch Labordiagnostik keine Zeit verloren gehen. Sind im initialen EKG ST-Streckensenkungen zu sehen, so gehört dieser Patient zur Hochrisikogruppe, und eine stationäre Abklärung ist erforderlich.

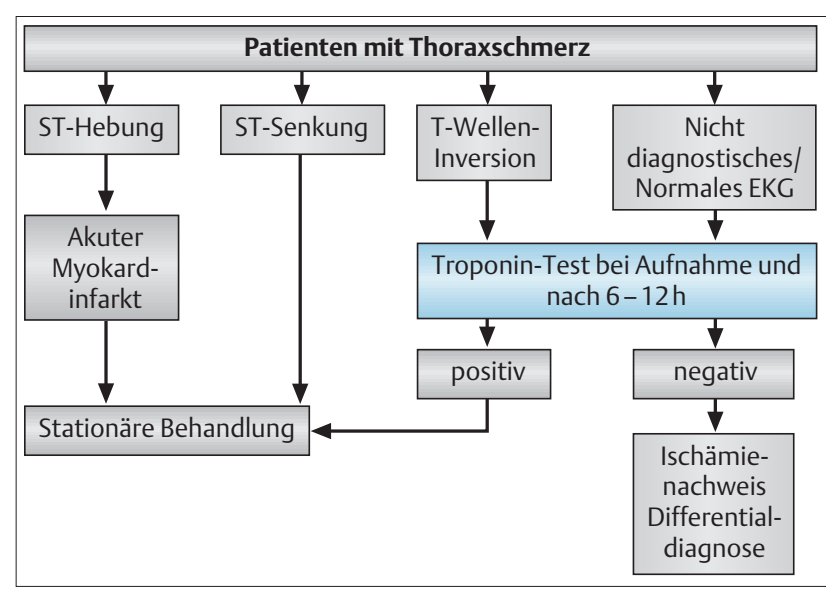

Abb.2 Vereinfachter Algorithmus zur Risikostratifizierung von Patienten mit akutem Thoraxschmerz.

Bei den übrigen Patienten stellt die Troponinbestimmung heute das Standardverfahren der weiteren Evaluierung dar. Bei positivem Troponin-Test handelt es sich ebenfalls um einen Hochrisikopatienten, der einer weiteren stationären Behandlung bedarf. Ein negativer Troponin-Test bei Aufnahme und nach 6-12 Stunden bedeutet kein erhöhtes akutes Risiko für den Patienten. Eine KHK ist damit aber nicht ausgeschlossen. Eine weitere stationäre Behandlung ist oft nicht erforderlich, und die Abklärung kann ambulant erfolgen, sofern andere lebensbedrohliche Erkrankungen (z.B. disseziertes Aortenaneurysma, Lungenembolie) ausgeschlossen sind (Abb.2). Darüber hinaus sind Patienten mit Kreislaufinstabilität, mit ventrikulären Herzrhythmusstörungen, mit Postinfarktangina und Diabetiker als Hochrisikopatienten einzustufen.

\section{kurzgefasst: Bei Vorliegen folgender Faktoren sind} Patienten mit ACS als Hochrisikopatienten einzustufen: ST-Streckensenkungen im Ruhe-EKG, Troponinerhöhung, Diabetes mellitus, Kreislaufinstabilität, Ventrikuläre Herzrhythmusstörungen (Kammertachykardien/ Kammerflimmern), Postinfarkt-Angina.

Autorenerklärung: Die Autoren erklären, dass sie keine finanziellen Verbindungen mit einer Firma haben, deren Produkt in dem Beitrag eine wichtige Rolle spielt (oder mit einer Firma, die ein Konkurrenzprodukt vertreibt).

\section{Literatur}

1 Alpert JS, Thygesen K, Antman E, Bassand JP. Myocardial infarction redefined. J Am Coll Cardiol 2000; 36: 959-969

2 Bertrand M, Simoons M, Keith AA et al. Management of acute coronary syndromes in patients presenting without persistent ST-segment elevation. Eur

3 Fox KA, Goodman SG, Klein W et al. Management of acute coronary syndroFox KA, Goodman SG, Klein W et al. Management of acute coronary syndro-
mes. Variations in practice and outcome. Eur Heart J 2002; 23: 1177-1189

4 mes. Variations in practice and outcome. Eur Heart J 2002; 23: 1177-1189 ents with acute chest pain by means of rapid testing for cardiac troponin T or ents with acute chest pain by means of rapid testing
troponin I. N Engl J Med 1997; 337: 1648-1653

5 Hamm CW, Ravkilde J, Gerhardt W et al. The prognostic value of serum troHamm CW, Ravkilde J, Gerhardt W et al. The prognostic value

ponin T in unstable angina. N Engl J Med 1992; 327: 146-150 Hasdai D, Behar S, Wallentin L et al. A prospective survey of the characteristics, treatments and outcomes of patients with acute coronary syndromes in Europe and the Mediterranean basin. Eur Heart J 2002; 23: 1190-1201

Heeschen C, Hamm C, Bruemmer J, Simoons ML. Predictive value of C-reactive protein and troponin T in patients with unstable angina. CAPTURE Investigators. Chimeric c7E3 AntiPlatelet Therapy in Unstable angina Refractory to standard treatment trial. J Am Coll Cardiol 2000; 35: 1535-1542

Mueller C, Buettner $\mathrm{H}$ et al. Inflammation and long-term mortality after nonST elevation acute coronary syndrome treated with a very early invasive strategy in 1042 consecutive patients. Circulation 2002; 105: 1412-1415

TIMI IIIb Investigators. Effects of tissue plasminogen activator and a comparison of early invasive and conservative strategies in unstable angina and non-Q-wave myocardial infarction. Circulation 1994; 89: 1545-1556 